J. Garrido, F. Vázquez, F. Morilla, and T. Hägglund, Practical advantages of inverted decoupling, Proceedings of the Institution of Mechanical Engineers, Part I: Journal of Systems and Control Engineering November 2011 Volume 225, Issue 7, pp. 977-992, ISSN 0959-6518, (http://pii.sagepub.com/content/225/7/977)

\title{
PRACTICAL ADVANTAGES OF INVERTED DECOUPLING
}

\author{
Juan Garrido $^{\mathrm{a}^{*}}$, Francisco Vázquez ${ }^{\mathrm{a}}$, Fernando Morilla ${ }^{\mathrm{b}}$, Tore Hägglund ${ }^{\mathrm{c}}$ \\ ${ }^{a}$ Department of Computer Science and Numerical Analysis, University of Córdoba, \\ Campus de Rabanales, 14071, Córdoba, Spain \\ *Fax: (+34)957218729; e-mail: juan.garrido@uco.es \\ ${ }^{b}$ Department of Computer Science and Automatic Control, UNED, Juan del Rosal 16, \\ 28040, Madrid, Spain \\ ${ }^{c}$ Department of Automatic Control, Lund University, Box 118, S-22100, Lund, Sweden
}

\begin{abstract}
This paper presents a study of the main advantages of inverted decoupling in $2 \times 2$ processes. Two simulation examples and an experimental process are used to show these advantages in comparison with simplified decoupling. The study is focused on the following practical advantages: the apparent process is the same as that obtained if one loop changes to manual, bumpless transfer and anti-windup are achieved easily using a feedforward input in the controllers, and abnormalities of secondary loops do not affect the opposite loop. Thanks to them, inverted decoupling may be a good and easy way to improve the performance of industrial TITO processes with interaction problems (when it can be applied).
\end{abstract}


Keywords: decoupling control, inverted decoupling, simplified decoupling, PID controller, bumpless transfer, anti-windup, TITO processes.

\section{1.- Introduction}

Most industrial processes are complex and multivariable in essence [1]. They consist of several measurement and control signals, and there are often complicated couplings between them, which can cause difficulties in feedback controller design. Process control problems are traditionally solved using single-loop PID controllers that are connected through well-known couplings such as cascade control, feed-forward control, ratio control, etc. These decentralized approaches have traditionally evolved through years of experience, and they are adequate when the interactions in different channels of the process are modest. Nevertheless, when interactions are significant, the decoupling is often treated inefficiently, e.g., by detuning control loops. Commonly, the most important loop is tuned to give good performance, while the other loops are detuned in such a way that the interaction with the first loop becomes acceptable. In these cases, a full matrix controller (centralized control) is advisable.

There are two approaches of centralized control: a pure centralized strategy [2-6] or a decoupling network combined with a diagonal decentralized controller [7-10]. In the last case, a decoupler is used to minimize interaction or to make the system diagonal dominant; then, the controllers are designed using some decentralized method. The decouplers combined with the single-loop controllers constitute the multivariable controller. 
Over the years decoupling has been addressed in the literature [11-14]. Some approaches are static [15], and others are dynamic [9-10], [14], [16]. Static decoupling guarantees complete decoupling only for low frequencies. This might not be enough to achieve good performance. Further, cross couplings at other frequencies must be handled in some way during controller design. Dynamic decoupling eliminates or reduces the interactions in a wider range of frequencies, but the design is more difficult and the decoupler elements are more complex and difficult to implement.

In addition, most of these methodologies focus on systems with two inputs and two outputs (TITO systems). They are one of the most prevalent categories of multivariable systems because there are real processes of this nature or because a complex process can be decomposed in 2x2 blocks with non negligible interactions between its inputs and outputs [9-10], [17-18].

Most decoupling approaches use a conventional decoupling scheme in which the process inputs are derived by a time-weighted combination of feedback controller outputs (Figure 1a). The most extended forms of conventional decoupling were termed ideal and simplified decoupling in [7]. This approach has received considerable attention in both control theory and industrial practice for several decades. However, this scheme presents several implementation problems that many companies are not willing to face up to; sometimes because of the costs of implementation or because it is necessary to make important changes in the control system.

\section{INSERT HERE FIGURE 1}

An alternative means of decoupling, called inverted decoupling, derives a process input as a time-weighted combination of one feedback controller output and the other process 
inputs. It is rarely mentioned in the literature, but it is very easy to implement when it can be applied [19]. Inverted decoupling scheme for a 2x2 process is shown in Figure $1 b$.

In [8], inverted decoupling is shown to be a form of ideal decoupling which can be implemented using the simple elements of simplified decoupling. In [20], a comparative study of simplified, ideal and inverted decoupling is presented. It is demonstrated that robust performance and robust stability of a nominally stable control system are equivalent for the three decoupling methods when the controllers are tuned to obtain identical nominal performance. Chen et al. [21] improved upon the inverted decoupling technique for a class of stable linear multivariable processes with multiple time delays and non-minimum-phase zeros. The main practical advantages of inverted decoupling over both ideal and simplified conventional decouplings are mentioned in them; however, they are not studied or exemplified in great detail.

In this work, the main practical advantages of inverted decoupling of 2x2 processes are explained and illustrated using several examples in comparison with simplified decoupling, in such a way that they can be clearly appreciated. The paper is structured as follows. Section 2 presents the equations of inverted and simplified decoupling, realizability problems, stability conditions and a brief comparison of both decouplings. In Section 3, the different processes used to highlight the advantages of inverted decoupling are described. Section 4 shows these practical advantages using two simulation examples and a real quadruple tank process. Finally, conclusions are summarized in Section 5. 


\section{2.- Inverted decoupling and conventional decoupling for TITO processes}

\section{1.- Decoupler design}

A decentralized control system with a decoupling matrix can be designed by combining a diagonal controller $\mathbf{C}(\mathrm{s})$ with a block compensator $\mathbf{D}(\mathrm{s})$ in such a way that the controller sees the apparent process $\mathbf{G}(\mathrm{s}) \cdot \mathbf{D}(\mathrm{s})$ as a set of $n$ completely independent processes. The essence of decoupling is the imposition of a calculation net that cancels the existing process interaction, allowing for independent control of the loops. In this way, as the open loop matrix $\mathbf{G}(\mathrm{s}) \cdot \mathbf{D}(\mathrm{s}) \cdot \mathbf{C}(\mathrm{s})$ is diagonal, the closed loop matrix $\mathbf{G}(\mathrm{s}) \cdot \mathbf{D}(\mathrm{s}) \cdot \mathbf{C}(\mathrm{s}) \cdot[\mathbf{I}+\mathbf{G}(\mathrm{s}) \cdot \mathbf{D}(\mathrm{s}) \cdot \mathbf{C}(\mathrm{s})]^{-1}$ is decoupled too.

For TITO processes, using the structure of conventional decoupling (Figure 1a), the design of the decoupler network is obtained from (1), generally specifying two elements of the decoupler $\mathbf{D}(\mathrm{s})$ or the two desired transfer functions of the apparent process $\mathbf{Q}(\mathrm{s})$. Two of the most extended forms are ideal decoupling and simplified decoupling.

$$
\mathbf{D}(\mathrm{s})=\mathbf{G}^{-1}(\mathrm{~s}) \cdot \mathbf{Q}(\mathrm{s})
$$

In ideal decoupling, the goal is to make the apparent processes as simple as the diagonal

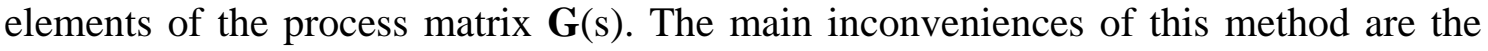
complexity of the decoupler elements (2) and realizability problems.

$$
\begin{gathered}
\mathbf{Q}(\mathrm{s})=\left(\begin{array}{cc}
g_{11}(\mathrm{~s}) & 0 \\
0 & g_{22}(\mathrm{~s})
\end{array}\right) \text { and } \\
\mathbf{D}(\mathrm{s})=\frac{\left(\begin{array}{cc}
g_{22}(\mathrm{~s}) \cdot g_{11}(\mathrm{~s}) & -g_{12}(\mathrm{~s}) \cdot g_{22}(\mathrm{~s}) \\
-g_{21}(\mathrm{~s}) \cdot g_{11}(\mathrm{~s}) & g_{11}(\mathrm{~s}) \cdot g_{22}(\mathrm{~s})
\end{array}\right)}{g_{11}(\mathrm{~s}) \cdot g_{22}(\mathrm{~s})-g_{12}(\mathrm{~s}) \cdot g_{21}(\mathrm{~s})}
\end{gathered}
$$

On the other hand, with simplified decoupling, two elements of the decoupler, generally the diagonal ones, are set to unity. Decoupler network design is easier; however the complexity of the apparent process is greater (3) 


$$
\begin{gathered}
\mathbf{D}(\mathrm{s})=\left(\begin{array}{cc}
1 & \frac{-g_{12}(\mathrm{~s})}{g_{11}(\mathrm{~s})} \\
\frac{-g_{21}(\mathrm{~s})}{g_{22}(\mathrm{~s})} & 1
\end{array}\right) \text { and } \\
\mathbf{Q}(\mathrm{s})=\left(\begin{array}{cc}
g_{11}(\mathrm{~s})-\frac{g_{21}(\mathrm{~s}) \cdot g_{12}(\mathrm{~s})}{g_{22}(\mathrm{~s})} & 0 \\
0 & g_{22}(\mathrm{~s})-\frac{g_{21}(\mathrm{~s}) \cdot g_{12}(\mathrm{~s})}{g_{11}(\mathrm{~s})}
\end{array}\right)
\end{gathered}
$$

Using the structure of inverted decoupling (Figure 1b), it is possible to keep the same apparent process $\mathbf{Q}$ (s) of ideal decoupling while using the simple decoupler elements of simplified decoupling (4), as it is demonstrated in [20].

$$
\begin{gathered}
d_{12}(\mathrm{~s})=\frac{-g_{12}(\mathrm{~s})}{g_{11}(\mathrm{~s})} \quad d_{21}(\mathrm{~s})=\frac{-g_{21}(\mathrm{~s})}{g_{22}(\mathrm{~s})} \\
\text { and } \quad \mathbf{Q}(\mathrm{s})=\left(\begin{array}{cc}
g_{11}(\mathrm{~s}) & 0 \\
0 & g_{22}(\mathrm{~s})
\end{array}\right)
\end{gathered}
$$

\section{2.- Stability of inverted decoupling}

If the expression of the whole decoupler $\mathbf{D}(\mathrm{s})$ is studied using the structure of the inverted decoupling configuration, the following equivalent expression of conventional decoupling is obtained

$$
\mathbf{D}(\mathrm{s})=\frac{1}{1-d_{12}(\mathrm{~s}) d_{21}(\mathrm{~s})}\left(\begin{array}{cc}
1 & d_{12}(\mathrm{~s}) \\
d_{21}(\mathrm{~s}) & 1
\end{array}\right)
$$

Using the decoupler elements in (4), it is proved that inverted decoupling is a different way to implement ideal decoupling in (2) with simpler decoupler elements. Assuming stable and realizable decoupler elements, the stability problem rests on the location of zeros in the equation (6), which is equivalent to the location of the zeros of the determinant of the transfer function matrix of the process. 


$$
\begin{aligned}
& 1-d_{12}(\mathrm{~s}) d_{21}(\mathrm{~s})=0 \\
& 1-\frac{-g_{12}(\mathrm{~s})}{g_{11}(\mathrm{~s})} \frac{-g_{21}(\mathrm{~s})}{g_{22}(\mathrm{~s})}=0 \\
& g_{11}(\mathrm{~s}) g_{22}(\mathrm{~s})-g_{12}(\mathrm{~s}) g_{21}(\mathrm{~s})=0
\end{aligned}
$$

Therefore, due to stability problems, the main disadvantage of inverted decoupling is the fact that it cannot be applied to processes with RHP zeros in the determinant of the transfer function matrix. For internal stability, these RHP zeros should appear in the apparent process. In a conventional decoupling structure, RHP zeros of the determinant of $\mathbf{G}(\mathrm{s})$ can be included in the desired equivalent open loop transfer functions $\mathbf{Q}(\mathrm{s})$. However, this is not possible using inverted decoupling. If the multivariable RHP zero is associated with a single output and is therefore included in the process transfer functions of the same row, inverted decoupling can be applied because the RHP zero will be cancelled.

Once the stability of the decoupler has been assured, internal stability of the closed loop system is verified if and only if all elements in the following matrix (7) have all their poles in the left-half plane and there is no RHP pole-zero cancellation in $\mathbf{G}(\mathrm{s}) \cdot \mathbf{K}(\mathrm{s})$ [12], where $\mathbf{S}(\mathrm{s})=[\mathbf{I}+\mathbf{G}(\mathrm{s}) \cdot \mathbf{D}(\mathrm{s}) \cdot \mathbf{C}(\mathrm{s})]^{-1}$ is the sensitivity transfer function matrix and $\mathbf{K}(\mathrm{s})=\mathbf{D}(\mathrm{s}) \cdot \mathbf{C}(\mathrm{s})$ represents the decentralized controller in series with the decoupler.

$$
\left[\begin{array}{cc}
(\mathbf{I}+\mathbf{K}(s) \cdot \mathbf{G}(s))^{-1} & -\mathbf{K}(s) \cdot \mathbf{S}(s) \\
\mathbf{S}(s) \mathbf{G}(s) & \mathbf{S}(s)
\end{array}\right]
$$

\section{3.- Realizability for inverted decoupling}

The realizability requirement for any decoupler is that all of its elements must be proper, causal and stable. For processes with time delays or non-minimum-phase zeros, direct calculation of the decoupler can lead to elements with prediction or right-halfplane (RHP) poles. Therefore, essential measures must be taken to deal with them. 
Besides the configuration of Figure 1b (configuration A), there is an alternative configuration (configuration B) in which the apparent process and decoupler elements are given by (8). These decoupler elements are the inverse of the decoupler elements of configuration A, and the apparent process is composed by the off diagonal elements of the process.

$$
\begin{array}{r}
d_{11}(\mathrm{~s})=\frac{-g_{11}(\mathrm{~s})}{g_{12}(\mathrm{~s})} \quad d_{22}(\mathrm{~s})=\frac{-g_{22}(\mathrm{~s})}{g_{21}(\mathrm{~s})} \\
\text { and } \mathbf{Q}(\mathrm{s})=\left(\begin{array}{cc}
g_{12}(\mathrm{~s}) & 0 \\
0 & g_{21}(\mathrm{~s})
\end{array}\right)
\end{array}
$$

When no configuration is realizable, it is necessary to add extra dynamics in a diagonal block $\mathbf{N}(\mathrm{s})$ between the system $\mathbf{G}(\mathrm{s})$ and the inverted decoupler $\mathbf{D}(\mathrm{s})$ in order to modify the process and to force the non-realizable elements into realizability. Then, inverted decoupling can be applied to the new process $\mathbf{G}_{\mathbf{N}}(\mathrm{s})=\mathbf{G}(\mathrm{s}) \cdot \mathbf{N}(\mathrm{s})$.

There are three aspects to take into account and to be inspected for each row to choose the proper configuration. Next, considering stable transfer functions $g_{i j}(\mathrm{~s})$ with time delays $\theta_{i j}$ and relative degrees $r_{i j}$, these aspects are studied separately:

1- Non-causal time delays must be avoided in decoupler elements. Using configuration A, according to (4), the time delays of the two decoupler elements would be $\theta_{1}=\theta_{12}-\theta_{11}$ and $\theta_{2}=\theta_{21}-\theta_{22}$. Using configuration $\mathrm{B}$, according to (8), the time delays of the decoupler elements would be the opposite ones. For realizability, the time delays of decoupler elements must be greater than or equal to zero. Therefore, the following cases can arise:

- $\theta_{1}$ and $\theta_{2}$ greater than or equal to zero: configuration A should be selected.

- $\theta_{1}$ and $\theta_{2}$ less than or equal to zero: configuration B should be selected.

- $\theta_{1}$ and $\theta_{2}$ equal to zero: any configuration can be selected. 
- $\theta_{1}$ and $\theta_{2}$ are different from zero and have opposite signs. In this case no configuration is initially realizable. In order to force realizability it is necessary to insert an additional time delay in one of the diagonal elements of $\mathbf{N}(\mathrm{s})$. Suppose the diagonal block of extra time delays $\mathbf{N}(\mathrm{s})=\operatorname{diag}\left(e^{-\theta_{n 1} s}, e^{-\theta_{n 2} s}\right)$ is proposed. Then, the new decoupler time delays would be given by (9) and they should be greater than or equal to zero for realizability.

$$
\begin{aligned}
& \theta_{1}^{N}=\theta_{1}-\theta_{n 1}+\theta_{n 2} \\
& \theta_{2}^{N}=\theta_{2}+\theta_{n 1}-\theta_{n 2}
\end{aligned}
$$

Since, generally, it is preferable to add the minimum extra dynamics, the additional time delay is the minimum absolute value between the absolute values of $\theta_{1}$ and $\theta_{2}$ (the other additional time delay will be equal to zero). Inserting this time delay in the proper input, the elements of the corresponding row of the new process $\mathbf{G}_{\mathbf{N}}(\mathrm{s})$ will have the same time delays and therefore, the time delay $\theta_{i}^{N}$ of one of the new decoupler elements will be equal to zero. That corresponds to the first or second situation of the previous cases, which would indicate us the adequate configuration.

Thus, if this minimum time delay $\theta_{i}$ is positive, the additional time delay must be inserted in the $i$-th diagonal element of $\mathbf{N}(\mathrm{s})$, that is, $\theta_{n i}$ in (9) should be equal to $\theta_{i}$ and the other extra time delay should be zero. In this way, as $\theta_{n i}$ in (9) has negative sign, the new decoupler time delay $\theta_{i}^{N}$ will be zero. The other decoupler time delay will be negative, because initially $\theta_{1}$ and $\theta_{2}$ have opposite signs and the absolute magnitude of $\theta_{i}$ is smaller. Then, the second previous case arises and configuration B is realizable.

On the other hand, if this minimum time delay $\theta_{i}$ is negative, the additional time delay must be inserted in the alternative diagonal element of $\mathbf{N}(\mathrm{s})$, because it has positive sign in (9). The time delay of the $i$-th diagonal element of $\mathbf{N}(\mathrm{s})$ should be zero. In this way, 
the new decoupler time delay $\theta_{i}^{N}$ will be zero. The other decoupler time delay will be positive, because initially $\theta_{1}$ and $\theta_{2}$ have opposite signs and the absolute magnitude of $\theta_{i}$ is smaller. Then, the first previous case arises and configuration A is realizable.

2- Decoupler elements must be proper. Similar to the analysis of time delays, using configuration A and according to (4), the relative degree of the two decoupler elements would be $r_{1}=r_{12}-r_{11}$ and $r_{2}=r_{21}-r_{22}$. Using configuration $\mathrm{B}$, according to (8), the relative degrees of the decoupler elements would be the opposite ones. For realizability, the relative degree of decoupler elements must be greater than or equal to zero. Therefore, the following cases can arise:

- $r_{1}$ and $r_{2}$ greater than or equal to zero: configuration A should be selected.

- $r_{1}$ and $r_{2}$ less than or equal to zero: configuration B should be selected.

- $r_{1}$ and $r_{2}$ equal to zero: any configuration can be selected.

- $r_{1}$ and $r_{2}$ are different from zero and have opposite signs. In this case no configuration is initially realizable. In order to force realizability it is necessary to insert in $\mathbf{N}(\mathrm{s})$ a simple stable pole $1 / \lambda$ with the adequate multiplicity $r_{n i}$ as follows:

$$
\frac{1}{(\lambda s+1)^{r_{i}}}
$$

Following the same previous procedure for time delays, we obtain that the minimum multiplicity $r_{n i}$ of the extra pole in (10) is the minimum absolute value between the absolute values of $r_{1}$ and $r_{2}$. Inserting this element in the proper input, the elements of the corresponding row of the new process $\mathbf{G}_{\mathbf{N}}(\mathrm{s})$ will have the same relative degree and therefore, the relative degree of one of the new decoupler elements will be equal to zero. Thus, if this minimum relative degree $r_{i}$ is positive, the additional dynamics must be inserted in the $i$-th diagonal element of $\mathbf{N}(\mathrm{s})$, and configuration $\mathrm{B}$ will be realizable. On the other hand, if this minimum relative degree $r_{i}$ is negative, the additional extra 
dynamics must be inserted in the alternative diagonal element of $\mathbf{N}(\mathrm{s})$ and configuration A will be realizable.

3- Decoupler elements with RHP poles must be avoided. When some transfer function $g_{i j}(\mathrm{~s})$ has a RHP zero, the configuration in which this $g_{i j}(\mathrm{~s})$ appears in the denominator of some decoupler element must be avoid, because this zero will become a RHP pole. Thus, the configuration with the decoupler element $d_{i j}(\mathrm{~s})$ should be selected. A special case that makes impossible the realizability of inverted decoupling appears when each one of the element of a same row has a different RHP zero. In this case, one of these zeros will always become an unstable pole in some decoupler element of any configuration, and therefore, no configuration is realizable.

However, when the same RHP zero appears in all elements of the same row of $\mathbf{G}(\mathrm{s})$, it is necessary to check the zero multiplicity $\eta_{i j}$ in each element. Similar to the analysis of time delays and relatives degrees, using configuration A and according to (4), the zero multiplicity in the two decoupler elements would be $\eta_{1}=\eta_{12}-\eta_{11}$ and $\eta_{2}=\eta_{21}-\eta_{22}$. Using configuration B, according to (8), the multiplicities in the decoupler elements would be the opposite ones. For realizability, the zero multiplicity in decoupler elements must be greater than or equal to zero. Therefore, the following cases can arise:

- $\eta_{1}$ and $\eta_{2}$ greater than or equal to zero: configuration A should be selected.

- $\eta_{1}$ and $\eta_{2}$ less than or equal to zero: configuration B should be selected.

- $\eta_{1}$ and $\eta_{2}$ equal to zero: any configuration can be selected.

- $\eta_{1}$ and $\eta_{2}$ are different from zero and have opposite signs. In this case no configuration is initially realizable. In order to force realizability it is necessary to insert in $\mathbf{N}(\mathrm{s})$ an element as follows: 
$\left(\frac{-s+z}{s+z^{*}}\right)^{\eta_{n i}}$

where $z^{*}$ is the complex conjugate of $z$, and $\eta_{n i}$ is the adequate multiplicity. Note that the relative degree of (11) is zero, and therefore, it does not affect to the previous conditions about relative degrees. Again, following the same previous procedure for time delays and relative degrees, we obtain that the minimum zero multiplicity $\eta_{n i}$ of the extra element in (11) is the minimum absolute value between the absolute values of $\eta_{1}$ and $\eta_{2}$. Inserting this element in the proper input, the elements of the corresponding row of the new process $\mathbf{G}_{\mathbf{N}}(\mathrm{s})$ will have the same zero multiplicity and therefore, the zero multiplicity of one of the new decoupler elements will be equal to zero. Thus, if this minimum zero multiplicity $\eta_{i}$ is positive, the additional dynamics must be inserted in the $i$-th diagonal element of $\mathbf{N}(\mathrm{s})$, and configuration B will be realizable. On the other hand, if this minimum zero multiplicity $\eta_{i}$ is negative, the additional extra dynamics must be inserted in the alternative diagonal element of $\mathbf{N}(\mathrm{s})$ and configuration A will be realizable.

For illustration, considering the following example [4]

$\mathbf{G}(s)=\left(\begin{array}{cc}\frac{e^{-2 s}}{s+2} & \frac{-e^{-6 s}}{s+2} \\ \frac{(s-0.5) \cdot e^{-3 s}}{(s+2)^{2}} & \frac{(s-0.5)^{2} \cdot e^{-8 s}}{2(s+2)^{3}}\end{array}\right)$

This process (12) has a multivariable RHP zero at $s=0.5$. Nevertheless, it is associated with a single output, the second one, and therefore, inverted decoupling can be applied. However, this RHP zero appears in the two process transfer functions of the second row with different multiplicity. According to the previous RHP zero conditions, the configuration B should be selected because $\eta_{1}=0$ and $\eta_{2}=-1$ (the second case of the previous analysis). However, according to the time delay conditions, no configuration is 
realizable because $\theta_{1}=4$ and $\theta_{2}=-5$, they have different signs. Thus, in order to achieve realizability and according to the previous time delay analysis, an extra time delay of 4 units has to be added in the first input, because the absolute value of $\theta_{1}$ is the smallest one, and it is positive.

In this case, the new process to be decoupled is given by (13), and configuration B is realizable. Then, using this configuration and according to (8), the decoupler elements are given by (14) and the apparent decoupled process is composed by off diagonal elements of (13). The RHP zero appears in the apparent process of the second output, which is necessary for internal stability.

$$
\begin{aligned}
& \left(\begin{array}{cc}
\frac{e^{-6 s}}{s+2} & \frac{-e^{-6 s}}{s+2} \\
\frac{(s-0.5) \cdot e^{-7 s}}{(s+2)^{2}} & \frac{(s-0.5)^{2} \cdot e^{-8 s}}{2(s+2)^{3}}
\end{array}\right) \\
& d_{11}(s)=1 \quad d_{22}(s)=\frac{-0.5(s-0.5) \cdot e^{-s}}{(s+2)}
\end{aligned}
$$

\section{4.- Advantages of inverted decoupling}

In spite of the fact that inverted decoupling can not be applied to process with RHP zeros in the determinant, it has many advantages compared to conventional decoupling. Some of them are related to implementation aspects, and they will be treated later. Furthermore, there are some fundamental advantages:

- The apparent processes do not contain sums of transfer functions, and therefore, tuning of the diagonal controllers is easier. In multivariable processes with strong cross couplings, even if the elements of the system have simple dynamics, decoupling may 
result in complicated diagonal apparent processes consisting of parallel coupled processes that may have different signs and different time delays [22]. An example of such a diagonal element is:

$G(s)=\frac{1.2}{(0.5 s+1)(0.7 s+1)} e^{-4 s}-\frac{1}{(3 s+1)(2 s+1)} e^{-s}$

which consists of a difference between two fairly simple transfer functions. If PID controllers are used to control a system with diagonal elements like (15), design methods that rely on simple process dynamics, like step response methods [23], are not appropriate.

- Decoupler elements do not contain either sum of transfer functions, so they are easy to design. Using conventional decoupling, in some cases, it is possible to have decoupler elements with complexity similar to that of process (15), and therefore, they are difficult to implement.

In addition, inverted decoupling also has several practical advantages over conventional decoupling that make this decoupling structure very interesting from an implementation point of view. These advantages [8] are the following:

- The apparent process seen by each controller, when inverted decoupling is implemented, is the same as that obtained if there was no decoupling and the alternate controller were in the manual mode.

- Inverted decoupling can often be implemented within a DCS using PID function blocks with feedforward inputs. This will automatically provide such features as initialization and bumpless transfer between manual and automatic.

- The antireset windup feature of the PID, combined with its feedforward input, can be used to directly take into account the saturation of the manipulated variables when inverted decoupling is implemented. 
- When decoupler outputs are used as cascade setpoints to lower level controllers, each decoupled control loop is immune to abnormalities (e.g., a valve at a limit or a secondary controller in manual) in the secondary of the opposite control loop.

Although this work focuses on TITO processes, these advantages can be also extended to higher dimensional systems because the complexity of the decoupler elements does not increase.

\section{3.- Process examples}

In order to study the previous practical advantages in more detail and in order to present a comparison between inverted decoupling and simplified decoupling, three TITO processes are used for this goal. Two of them are simulation processes and the other one is an experimental system. They are multivariable processes with directionality problems and great interactions, and therefore, the use of a decoupling control structure is justified in all of them. The decoupler elements calculated for each process are the same in both decoupling structures (inverted and simplified). A diagonal controller is tuned for each decoupling structure in order to obtain the same performance. The diagonal control consists of two PI controllers that are tuned in the frequency domain using phase margins or gain margins as performance specifications [24].

The simulation processes are the Wood and Berry binary distillation column and the Niederlinski process. The Wood and Berry process [25] is

$$
\boldsymbol{G}_{\mathrm{WB}}(s)=\left(\begin{array}{cc}
\frac{12.8 \times \mathrm{e}^{-\mathrm{s}}}{16.7 \mathrm{~s}+1} & \frac{-18.9 \times \mathrm{e}^{-3 \mathrm{~s}}}{21 \mathrm{~s}+1} \\
\frac{6.6 \times \mathrm{e}^{-7 \mathrm{~s}}}{10.9 \mathrm{~s}+1} & \frac{-19.4 \times \mathrm{e}^{-3 \mathrm{~s}}}{14.4 \mathrm{~s}+1}
\end{array}\right)
$$


Two PI controllers are tuned to achieve a phase margin of $60^{\circ}$ and a gain margin of 4 in both loops. The PI parameters are shown in Table 1. The decoupler elements are:

$$
d_{12}(s)=\frac{1.1742 \cdot(\mathrm{s}+0.05988)}{\mathrm{s}+0.04762} \cdot \mathrm{e}^{-2 \cdot \mathrm{s}} \quad d_{21}(\mathrm{~s})=0.44945 \cdot \frac{(\mathrm{s}+0.06944)}{\mathrm{s}+0.09174} \cdot \mathrm{e}^{-4 \cdot \mathrm{s}}
$$

INSERT HERE TABLE 1

The Niederlinski process used for simulation is:

$$
\boldsymbol{G}_{\mathrm{N}}(\mathrm{s})=\frac{1}{(0.1 \mathrm{~s}+1)(0.2 \mathrm{~s}+1)^{2}}\left(\begin{array}{cc}
-1 & \frac{0.5}{0.1 \mathrm{~s}+1} \\
\frac{2.4}{0.5 \mathrm{~s}+1} & 1
\end{array}\right)
$$

Two PI controllers are tuned to achieve a phase margin of $60^{\circ}$ in both loops. The PI parameters are listed in Table 2. The decoupler elements are:

$$
d_{12}(\mathrm{~s})=\frac{5}{\mathrm{~s}+10} \quad d_{21}(\mathrm{~s})=\frac{-4.8}{\mathrm{~s}+2}
$$

\section{INSERT HERE TABLE 2}

The experimental process is a quadruple tank plant [26] of the lab of the department of Automatic Control of Lund University. It was configured in order to have interaction problems without multivariable RHP zeros, and then it was identified. The resultant model is

$$
\boldsymbol{G}_{\mathrm{T}}(\mathrm{s})=\left(\begin{array}{cc}
\frac{1.4}{14.62 \cdot s+1} & \frac{0.97}{(12.52 \cdot s+1) \cdot(8.63 \cdot s+1)} \\
\frac{1.09}{(11.96 \cdot s+1) \cdot(9.26 \cdot s+1)} & \frac{1.15}{13.7 \cdot s+1}
\end{array}\right)
$$

Input and output units in (18) are in $\mathrm{V}$, in the range of [0-10] V. The outputs are the level of the lower tanks inside the range of [0-20] $\mathrm{cm}$, and the inputs are flow references, [0-100] \%, to the secondary control loops that regulate the operation of the pumps. The identification was carried out around the operation point with the output $\boldsymbol{y}=[7,6.5] \mathrm{V}$ (or $[14,13] \mathrm{cm})$ and the input $\boldsymbol{u}=[6,6] \mathrm{V}$. 
Two PI controllers were tuned to obtain a phase margin of $60^{\circ}$ in both loops limiting the bandwidth frequency around $0.1 \mathrm{rad} / \mathrm{s}$, where the interactions problems of the process are greater. The PI parameters are listed in Table 3. The decoupler elements are:

$$
d_{12}(\mathrm{~s})=\frac{-0.6929 \cdot(14.62 \cdot \mathrm{s}+1)}{(12.52 \cdot \mathrm{s}+1) \cdot(8.63 \cdot \mathrm{s}+1)} \quad d_{21}(\mathrm{~s})=\frac{-0.9478 \cdot(13.7 \cdot \mathrm{s}+1)}{(9.26 \cdot \mathrm{s}+1) \cdot(11.96 \cdot \mathrm{s}+1)}
$$

\section{INSERT HERE TABLE 3}

\section{4.- Practical advantages of inverted decoupling}

\section{1.- The apparent process}

“The apparent process seen by each controller, when inverted decoupling is implemented, is the same as that obtained if there was no decoupling and the alternate controller was in manual mode.”

For instance, if the element $d_{12}$ is disabled in the inverted decoupling scheme of Figure 1b, although both loops are in automatic mode, the second loop remains decoupled while the first one not. The control scheme of the second loop is similar to a feedforward control combined with a feedback loop ( $A_{F F}$ mode). Thus, the apparent process for the second controller is $g_{22}(\mathrm{~s})$ (using both inverted and simplified decoupling). When the first loop is decoupled, using inverted decoupling, the apparent process is the same, $g_{22}(\mathrm{~s})$. However, using simplified decoupling, the apparent process changes; in this case, it is $g_{22}(\mathrm{~s})-\left(g_{12}(\mathrm{~s}) \cdot g_{21}(\mathrm{~s})\right) / g_{11}(\mathrm{~s})$. Consequently, if the same PID parameters are used, the performance of this output will be affected depending on the state (decoupled or not) of the first loop. In order to maintain the same performance, it would be necessary to change the PID parameters. 
Using inverted decoupling and the same PID parameters, the performance of the decoupled output is the same because the apparent process does not change. Nevertheless, the response of the other loop can deteriorate due to the interactions; it depends on the specific process to control and its interactions.

However, there are TITO processes in which the PID controllers of a decentralized control are tuned taking only into account the dynamics of the diagonal elements of the process, e.g., $g_{11}(\mathrm{~s})$ and $g_{22}(\mathrm{~s})$, and therefore, the achieved performance is poor due to interaction effects. In these cases, the system response could be improved easily adding the two decoupling elements of inverted decoupling (if the system determinant do not have RHP zeros). The PID parameters do not need to be changed, and it would be very simple to return to the old control configuration if the new one presents any problem.

In the following example, the Wood and Berry process is controlled keeping decoupled only one output and both control loops in automatic mode, in order to show how the apparent process changes using simplified decoupling and it remains the same using the inverted decoupling. In Figure 2, the output and input responses of the Wood and Berry process are shown when the following experiment is carried out with both decoupling structures. First, both control loops are in decoupled automatic mode and there is a unit step change in the second reference. There are not interactions in the first loop, and both decoupling schemes have similar performance. At $t=100 \mathrm{~min}$, the element $d_{12}$ is disconnected, and therefore, the first control loop is not decoupled (its mode changes from automatic mode with decoupling to automatic mode without it, $A_{F F^{-}}>A$ ). At $t=200$ min, there is another unit step change in the second reference, and there is a transitory response in the first loop due to the process interactions. However, in this case, using 
simplified decoupling, the performance of the second loop is very different from that of the previous step change, when both control loops were decoupled.

In this simulated example, it is pointed out clearly that the apparent process of the second loop has changed, and if the same PID parameters are used, it shows a more oscillatory response. On the other hand, using inverted decoupling, the same performance is achieved when the decoupler element $d_{12}$ is disabled, and additionally, the interaction rejection seems to be better.

\section{INSERT HERE FIGURE 2}

In any case, it is not very usual to work in automatic mode keeping decoupled only one control loop, and alternating the other loop between decoupled mode and not decoupled mode. It is more common to work keeping in manual mode the not decoupled loop. Thus, only one loop is maintained in decoupled automatic mode, while the other control loop is in manual mode without decoupling (Figure 3). It is similar to a feedforward control problem.

\section{INSERT HERE FIGURE 3}

In Figure 4, the output and control signal responses of the Niederlinski process are shown when a very similar experiment as the previous one is carried out. In this case, both loops are initially in automatic mode with decoupling, and there is a unit step change in the second reference. Then, at $t=5 \mathrm{~s}$, the first loop changes to manual mode $\left(A_{F F^{-}}>M\right)$. At $t=10 \mathrm{~s}$, there is another unit step change in the second reference. Again, it can be appreciated that the performance of this loop in the second step change is different using simplified decoupling, because the apparent process changes and the PID parameters remain unchanged. Its response is much slower than that achieved in the first step change, and that obtained with inverted decoupling. In order to maintain the same 
performance in the second loop, before the second step change takes place, the PID parameters should be exchanged for those used with inverted decoupling

\section{INSERT HERE FIGURE 4}

\section{2.- Bumpless transfer}

"Inverted decoupling can often be implemented within a DCS using PID function blocks with feedforward inputs. This will automatically provide such features as initialization and bumpless transfer between manual and automatic.”

One of the main practical problems of using decoupling control is to assure bumpless transfer between the operation modes: manual $(M)$, automatic $(A)$ and automatic with decoupling $\left(A_{F F}\right)$. In the examples of the previous section, the mode changes have been carried out with bumpless transfer. However, the system response could become seriously deteriorated if this problem is not taken into account in the control implementation. For instance, Figure 5 shows an experiment carried out with and without bumpless transfer using inverted decoupling in the quadruple tank process.

Initially, the first loop is in manual mode and its output is $16.5 \mathrm{~cm}$. The second loop is in automatic mode without decoupling and its output is $15.5 \mathrm{~cm}$. At $t=50 \mathrm{~s}$, there is a step change in the first control signal, the first output changes and a transitory response appears in the second output due to the interactions. At $t=250 \mathrm{~s}$, the second loop changes from automatic mode without decoupling to automatic mode with decoupling. Without bumpless transfer, there is an important transient in both loops due to a sudden change in the second control signal. Then, at $t=450 \mathrm{~s}$, there is a negative step change in the first control signal; however, in this case, there is no transitory response in the second output due to interactions, because the second loop is working in automatic 
mode with decoupling. Finally, at $t=650 \mathrm{~s}$, the second loop changes to manual mode and again, without bumpless transfer, there is a new important transitory response. A similar response can be obtained using simplified decoupling.

\section{INSERT HERE FIGURE 5}

In Figure 6, it is shown the structure of a PID controller with feedforward input (FF) that solves the bumpless transfer problem using inverted decoupling. In order to achieve bumpless transfer, the controller uses a bias which is updated when the operation mode changes between working with feedforward action and without it. When the feedforward input is enabled (FF_on), the current value of the feedforward input is subtracted to the bias; when it is disenabled (FF_off), the current value of the feedforward input in that moment is added to the bias.

$$
\begin{aligned}
& F F \text { _on } \Rightarrow B=B-F F \\
& F F \_ \text {off } \Rightarrow B=B+F F
\end{aligned}
$$

\section{INSERT HERE FIGURE 6}

To carry out the update of the bias, the controller has a feedforward control unit (FFCU). This unit detects changes of operation mode through the binary input 'enableFF'. When this input changes, the feedforward action is enabled or disenabled and the bias is calculated according to the previous expressions (22). In order to achieve bumpless transfer in operation mode changes, both the PID and the manual control unit (MCU) have an integrator with tracking mode of the whole controller output [27]. Furthermore, these two blocks have an extra input connected to the output of the FFCU (bias plus feedforward input), in order to obtain bumpless transfer when the feedforward action is disenabled. The blocks of the PID controller in Figure 6 are depicted in more detail in Figure 7.

INSERT HERE FIGURE 7 
The block PID_FF is a PI-D controller with tracking mode and an extra input for the feedforward-bias input. The MCU has an integrator to track the output signal of the whole controller, and an extra input for the feedforward-bias action.

The possible inconveniency of this implementation is the fact that the FFCU needs some discontinuous blocks, like hit crossing blocks to update the bias. In order to avoid this, a good option could be to implement the controller in a discrete form. Another approach that easily takes care of bumpless transfer and windup problems is to implement the PID controller in incremental form. See [27].

Using simplified decoupling, the bumpless transfer problem can be solved using simplified decoupling as well. However, in this case, the FFCU must be placed between the decoupler network and the process, as it is shown in Figure 8. Nevertheless, in order to implement an anti-windup scheme in this configuration, extra blocks are necessary just before the process inputs. Using inverted decoupling, anti-windup implementation is achieved directly with the scheme of Figure 6, thanks to the saturation model and the tracking mode.

\section{INSERT HERE FIGURE 8}

Therefore, it is possible to achieve bumpless transfer using both inverted and simplified decoupling. They are only different in the PID parameter tuning and the apparent diagonal process. Nevertheless, the implementation is much easier using inverted decoupling than using simplified decoupling.

Note that the case of bumpless transfer problem when the PID parameters are modified has not been studied. However, with the PI-D configuration in Figure 7, there should not be transitory responses, as long as the reference is not weighted. 


\section{3.- Anti-windup}

“The antireset windup feature of the PID, combined with its feedforward input, can be used to directly take into account the saturation of the manipulated variables when inverted decoupling is implemented."

Using inverted decoupling with the PID controller of Figure 6, antireset windup feature is achieved directly. It can use the input saturation model inside the PID controller or a reset feedback input which receives the real measured process input. In this last case, there is no saturation model because the real manipulated signal is measured.

With simplified decoupling, there are several options to implement an anti-windup strategy. Nevertheless, it is not as easy as with inverted decoupling. In this paper, the scheme of Figure 8 is adopted [20]. It is necessary to measure the control action of the decoupler elements $d_{21}$ and $d_{12}$, as well as the real process inputs (or the estimated real inputs from input constraint models). Furthermore, the PID controllers need a reset signal input to work in tracking mode. This signal is obtained, subtracting from the saturated process input $u$ the factor of the bias and decoupling action $\left(B \_F F\right)$ from the FFCU (Figure 8). The saturated process input can be calculated implementing a saturation model, or measuring directly the real process input.

In Figure 9, the next experiment is carried out using inverted decoupling with and without anti-windup mechanisms. In order to show the windup problem, the first input is limited in the range of [0-5.75] V. The experiment starts with the references [15, 15.5] $\mathrm{cm}$ (or [7.5, 7.75] V) in each loop, respectively. At $t=50 \mathrm{~s}$, a $1.5 \mathrm{~cm}$ (or $0.75 \mathrm{~V}$ ) 
step change is carried out in the first reference, and then, at $t=600 \mathrm{~s}$, there is a similar step change in the second one.

When no anti-windup scheme is used, the second reference is lost because the directionality of the control signal vector is modified after the maximum limit of the first input signal is reached. And then, after the change of the second reference, there is a large transitory before it is reached. All these problems are avoided using anti-windup mechanisms. Similar results can be obtained using simplified decoupling; nevertheless, the implementation is more complex, as mentioned previously.

\section{INSERT HERE FIGURE 9}

\subsection{Immunity to abnormalities in secondary control loops}

"When decoupler outputs are used as cascade setpoints to lower level controllers, each decoupled control loop is immune to abnormalities (e.g., a valve at a limit or a secondary controller in manual) in the secondary of the opposite control loop.”

For instance, the tank process has two secondary loops for the flows provided by the pumps, and therefore, the measured flows can be fed back through the decoupler elements. If the dynamics of the secondary loops are considered fast, they can be neglected in the design of decoupler elements, although little interactions may appear. Nevertheless, the rejection of input disturbances is much better. On the other hand, if the dynamics of the secondary loops are non-negligible, they must be taken into account in the decoupler design, in order to avoid a great loss of decoupling.

Figure 10a shows the comparison between decoupling using and not using the measured flows of the secondary loops. Initially, the references are $[15,15.5] \mathrm{cm}$ (or $[7.5,7.75]$ 
$\mathrm{V}$ ) in each loop, respectively. At $t=50 \mathrm{~s}$, a $1.5 \mathrm{~cm}$ (or $0.75 \mathrm{~V}$ ) step change is carried out in the first reference, and then, at $t=850 \mathrm{~s}$, there is a similar one in the second reference.

When the measured flows are used for decoupling, there is some loss of decoupling performance in the first loop and the peak response is higher. The second loop has practically the same performance.

\section{INSERT HERE FIGURE 10}

Since the real input to the process is measured, inverted decoupling has another advantage over simplified decoupling when this signal is fed back for decoupling: each decoupled control loop is almost immune to input disturbances in the opposite control loop. That is shown in Figure 10b, where a unit step change is applied to each loop as an input disturbance. For instance, at $t=50 \mathrm{~s}$, the input disturbance in the first loop does not have almost effect in the response of the second loop. This cannot be achieved using simplified decoupling.

\section{5.- Loss of the practical advantages}

Sometimes, one of the elements $d_{12}$ or $d_{21}$ of the decoupler is not realizable because it has a positive time delay or the numerator degree is greater then the denominator degree. In this case, it can be necessary to add extra dynamics between the decoupler and the process. In that case, it is not correct to measure the controlled variable of the secondary loop or the real input of the process to feed back for decoupling, because it differs from the decoupler output. Therefore, the other control loop is not immune to abnormalities in the secondary or input disturbances in the opposite control loop. Although the apparent process seen by each controller, when inverted decoupling is 
implemented, changes as well, it is still the same as that obtained if there was no decoupling and the alternate controller was in manual mode.

If the PID controllers do not have feedforward input, the advantages of inverted decoupling, to implement anti-windup schemes and to solve the bumpless transfer problem, are lost. Then, these problems should be solved in the same way as for simplified decoupling.

\section{5.- Conclusions}

Inverted decoupling shows four important practical advantages over simplified decoupling that have been studied in this paper using several examples. In addition, practical results are presented since these advantages are exemplified and verified on a laboratory process.

If one loop changes to manual or automatic mode without decoupling, the other loop remains decoupled with the same performance because the apparent process is the same. Using simplified decoupling, this apparent process is different, and therefore, using the same PID parameters, the performance can change much or not, depending on the specific process.

The bumpless transfer and initialization problems are easily avoided using a feedforward input in the PID controllers. Although this problem can be solved using simplified decoupling as well, its implementation is much more complicated. 
It is very easy to implement anti-windup strategies using inverted decoupling because the PID limits can be used for the saturation model. In simplified decoupling, that is more difficult because the decoupler outputs are not the same as the PID controller outputs.

The abnormalities of secondary control loops do not affect the opposite loop. In addition, since the real input to the process can be measured, each decoupled control loop is almost immune to input disturbances in the other control loop. This is not the case when simplified decoupling is used, because the decoupler signals are generated from signals in previous points to the disturbance inputs. Note that care should be taken in the dynamics of the secondary loops. If they are not very fast in comparison with system dynamics, inverted decoupling can lose some decoupling performance and obtain a worse response. In addition, note that some of these advantages can be lost due to realizability problems of the decoupler elements.

Many industrial TITO processes are controlled by decentralized PID controllers that were tuned without taking the interactions into account. As the apparent process is the same using inverted decoupling for such processes, their performance could be easily improved simply adding two decoupling blocks (lead-lags plus time delay blocks). This is important, because it means that inverted decoupling can be used in most modern DCS systems using the standard blocks that are already available in their function block library. Thanks to the easiness of implementation and achieving bumpless transfer, it would be easy to alternate between a decentralized or centralized control without modifying the PID parameters. For this reason, inverted decoupling may be a good and 
easy way to improve the performance of industrial TITO processes with interaction problems.

\section{Acknowledgments}

This work was supported by the Spanish CICYT under Grant DPI 2007-62052. This support is very gratefully acknowledged. Moreover, J. Garrido expresses appreciation for the FPU fellowship (Ref. AP2006-01049) from the Spanish Ministry of Education.

\section{References}

[1] Shinskey, F. C. Sistemas de control de procesos. Aplicación, diseño y sintonización. Tomo 1, 2006, (McGrawhill).

[2] Wang, Q. W., Zou, B., Lee, T., and Bi, Q. Auto-tuning of Multivariable PID Controllers from Decentralized Feedback. Automatica, 1997, 33(3) 319-330.

[3] Wang, Q. W., Zhang, Y., and Chiu, M. S. Decoupling internal model control for multivariable systems with multiple time delays. Chemical Engineering Science, 2002, 57, 115-124.

[4] Wang, Q. W., Zhang, Y., and Chiu, M. S. None-interacting control design for multivariable industrial processes. Journal of Process Control, 2003, 13(3) 253265.

[5] Morilla, F., Vázquez, F., and Garrido, J. Centralized PID Control by Decoupling for TITO Processes. Proceedings of 13th IEEE International Conference on Emerging Technologies and Factory Automation, Hamburg, 2008, pp. 1318-1325. 
[6] Garrido, J., Morilla, F., and Vázquez, F. Centralized PID Control by Decoupling of a Boiler-Turbine Unit. In Tenth European Control Conference, Budapest, 2009.

[7] Waller, K. Decoupling in distillation. AIChE Journal 1974, 20 (3) 592-594.

[8] Wade, H. L. Inverted decoupling: a neglected technique. ISA Transactions, 1997, 36(1) 3-10.

[9] Nordfeldt, P. PID control of TITO systems. Licenciate thesis ISRN LUTFD2/TFRT-3238-SE, Department of Automatic Control, Lund Institute of Technology, Sweden, December, 2005.

[10] Tavakoli, S., Griffin, I., and Fleming, P. J. Tuning of decentralised PI (PID) controllers for TITO processes. Control Engineering Practice, 2006, 14, 10691080.

[11] Maciejowski, J. Multivariable Feedback Design, 1989 (Addison-Wesley).

[12] Skogestad, S., and Postlethwaite, I. Multivariable Feedback Control: Analysis and Design, 1996 (John Wiley \& sons).

[13] Goodwin, G. C., Graebe, S. F., and Salgado, M. E. Control System Design, 2001 (Prentice-Hall).

[14] Wang, Q. W. Decoupling Control. Lecture Notes in Control and Information Sciences, 285, 2003 (Springer-Verlag).

[15] Åström, K. J., Johansson, K. H., and Wang, Q. W. Design of decoupled PI controller for two-by-two systems. IEE Proceedings on Control Theory and Applications, 2002, 149, 74-81.

[16] Vázquez, F., and Morilla, F. Tuning decentralized PID controllers for MIMO systems with decouplers. Proceeding of the 15th World Congress of the IFAC, Barcelona, Spain, 2002. 
[17] Vázquez, F., Morilla, F., and Dormido, S. An iterative method for tuning decentralized PID controllers. Proceeding of the $14^{\text {th }}$ IFAC World Congress, 1999, pp. 491-496.

[18] Vázquez, F. Diseño de controladores PID para sistemas MIMO con control descentralizado. PhD Thesis, UNED, 2001.

[19] Zheng, J., Guo, G., and Wang, Y. Feedforward decoupling control design for dual-actuator system in hard disk drives. IEEE Transactions on magnetics, 2004, 40(4), 2080-2082.

[20] Gagnon, E., Pomerleau, A., and Desbiens, A. Simplified, ideal or inverted decoupling? ISA Transactions, 1998, 37, 265-276.

[21] Chen, P., and Zhang, W. Improvement on an inverted decoupling technique for a class of stable linear multivariable processes. ISA Transactions, 2007, 46, 199210.

[22] Nordfeldt, P., and Hägglund, T. Decoupler and PID controller design of TITO systems. Journal of Process Control, 2006, 16, 923-936.

[23] Hägglund, T., and Åström, K. J. Revisiting the Ziegler-Nichols step response method for PID control. Journal of Process Control, 2004, 14 (6), 635-650.

[24] Morilla, F., and Dormido, S. Methodologies for the Tuning of PID Controllers in the frequency domain. Proceedings of PID’00. Tarrasa, 2000, pp.155-160.

[25] Wood, R. K., and Berry, M. W. Terminal composition control of a binary distillation column. Chemical Engineering Science,1973, 28.

[26] Johansson, K. H. The quadruple-tank process: a multivariable laboratory process with an adjustable zero. IEEE Transactions on Control System Technology, 2000, 8(3) 456-465. 
[27] Åström, K. J., and Hägglund, T. Advanced PID Control. ISA-The Instrumentation, Systems, and Automation Society, 2006.

\section{Appendix}

\section{Notation}

A automatic mode

$A_{F F} \quad$ automatic mode with decoupling

$B \quad$ (scalar) bias action (equation (22))

C(s) (transfer function matrix) diagonal controller

D(s) (transfer function matrix) decoupler network (equation (1))

$d_{11}$ (s) (scalar transfer function) element $(1,1)$ of $\mathbf{D}(\mathrm{s})$ (equation (8))

$d_{12}$ (s) (scalar transfer function) element $(1,2)$ of $\mathbf{D}$ (s) (equation (4))

$d_{21}$ (s) (scalar transfer function) element $(2,1)$ of $\mathbf{D}(\mathrm{s})$ (equation (4))

$d_{22}$ (s) (scalar transfer function) element $(2,2)$ of $\mathbf{D}(\mathrm{s})$ (equation (8))

FF (scalar) feedforward action (equation (22))

FF_off disenabling feedforward action (equation (22))

FF_on enabling feedforward action (equation (22))

G(s) (scalar transfer function) monovariable process (equation (15))

G(s) (transfer function matrix) multivariable process (equation (1))

$\mathbf{G}_{\mathrm{N}}(\mathrm{s})$ (transfer function matrix) multivariable Niederlinski process (equation (18))

$\mathbf{G}_{\mathrm{T}}(\mathrm{s})$ (transfer function matrix) multivariable quadruple tank process (equation (20))

$\mathbf{G}_{\mathrm{WB}}(\mathrm{s}) \quad$ (transfer function matrix) multivariable Wood \& Berry process (equation

$g_{11}(\mathrm{~s})$ (scalar transfer function) element $(1,1)$ of $\mathbf{G}(\mathrm{s})$ (equation (2))

$g_{12}(\mathrm{~s})$ (scalar transfer function) element $(1,2)$ of $\mathbf{G}(\mathrm{s})$ (equation (2))

$g_{21}(\mathrm{~s})$ (scalar transfer function) element $(2,1)$ of $\mathbf{G}(\mathrm{s})$ (equation (2)) 
$g_{22}(\mathrm{~s})$ (scalar transfer function) element $(2,2)$ of $\mathbf{G}(\mathrm{s})$ (equation (2))

K(s) (transfer function matrix) decentralized controller in series with the decoupler (equation (7))

M manual mode

$\mathbf{N}(s)$ (transfer function matrix) extra dynamics to force realizability

Q(s) (transfer function matrix) apparent process (equation (1))

$r_{i j} \quad$ (scalar) relative degree of the element $g_{\mathrm{ij}}(\mathrm{s})$ (equation (10))

S(s) (transfer function matrix) sensitivity function (equation (7))

$t \quad$ (scalar) time signal

u (vector) control signal

$\boldsymbol{y} \quad$ (vector) system output

Z (scalar) value of a zero RHP (equation (11))

$\lambda \quad$ (scalar) time constant of an extra pole (equation (10))

$\theta_{i j} \quad$ (scalar) time delay of the element $g_{\mathrm{ij}}(\mathrm{s})$

$\eta_{i j} \quad$ (scalar) RHP zero multiplicity of the element $g_{i j}(s)$ (equation (11)) 


\section{Tables}

\begin{tabular}{ccccc}
\hline Method & $\boldsymbol{K} \boldsymbol{p}_{\boldsymbol{I}}$ & $\boldsymbol{K} \boldsymbol{p}_{2}$ & $\boldsymbol{T \boldsymbol { i } _ { 1 }}$ & $\boldsymbol{T i}_{\boldsymbol{2}}$ \\
\hline Inverted & 0.491 & -0.095 & 8.673 & 11.107 \\
Simplified & 0.179 & -0.044 & 2.615 & 3.230 \\
\hline
\end{tabular}

Table 1 - PI parameters for the Wood and Berry process

\begin{tabular}{ccccc}
\hline Method & $\boldsymbol{K} \boldsymbol{p}_{\boldsymbol{1}}$ & $\boldsymbol{K} \boldsymbol{p}_{\boldsymbol{2}}$ & $\boldsymbol{T \boldsymbol { i } _ { \boldsymbol { 1 } }}$ & $\boldsymbol{\boldsymbol { T i } _ { \boldsymbol { 2 } }}$ \\
\hline Inverted & -1.207 & 1.207 & 0.423 & 0.423 \\
Simplified & -0.722 & 0.722 & 0.741 & 0.741 \\
\hline
\end{tabular}

Table 2 - PI parameters for the Niederlinski process

\begin{tabular}{ccccc}
\hline Method & $\boldsymbol{K} \boldsymbol{p}_{\boldsymbol{I}}$ & $\boldsymbol{K} \boldsymbol{p}_{\mathbf{2}}$ & $\boldsymbol{T \boldsymbol { i } _ { \boldsymbol { I } }}$ & $\boldsymbol{T i}_{\boldsymbol{2}}$ \\
\hline Inverted & 0.54 & 0.59 & 4.8 & 4.4 \\
Simplified & 0.57 & 0.61 & 2.5 & 2.3 \\
\hline
\end{tabular}

Table 3 - PI parameters for the quadruple tank process 


\section{Figure Captions}

Figure 1 - Decoupling control system of a TITO process: (a) conventional decoupling;

(b) inverted decoupling

Figure 2 - Wood and Berry process outputs and control signals (one output decoupled, both loops in automatic mode)

Figure 3 - One output decoupled, the alternate input in manual mode

Figure 4 - Niederlinski process outputs and control signals (one loop decoupled,

alternate loop in manual mode)

Figure 5 - Tank process outputs and control signals with and without bumpless transfer (inverted decoupling)

Figure 6 - PID control with feedforward input and bumpless transfer

Figure 7- Blocks of the PID controller with feedforward input

Figure 8 - Simplified decoupling with FFCU to get bumpless transfer

Figure 9 - Tank process outputs and control signals with and without anti-windup

(inverted decoupling)

Figure 10 - Tank process outputs and control signals using and not using the measured flows of secondary loops in the inverted decoupling scheme: (a) tracking references, (b) rejecting disturbances 


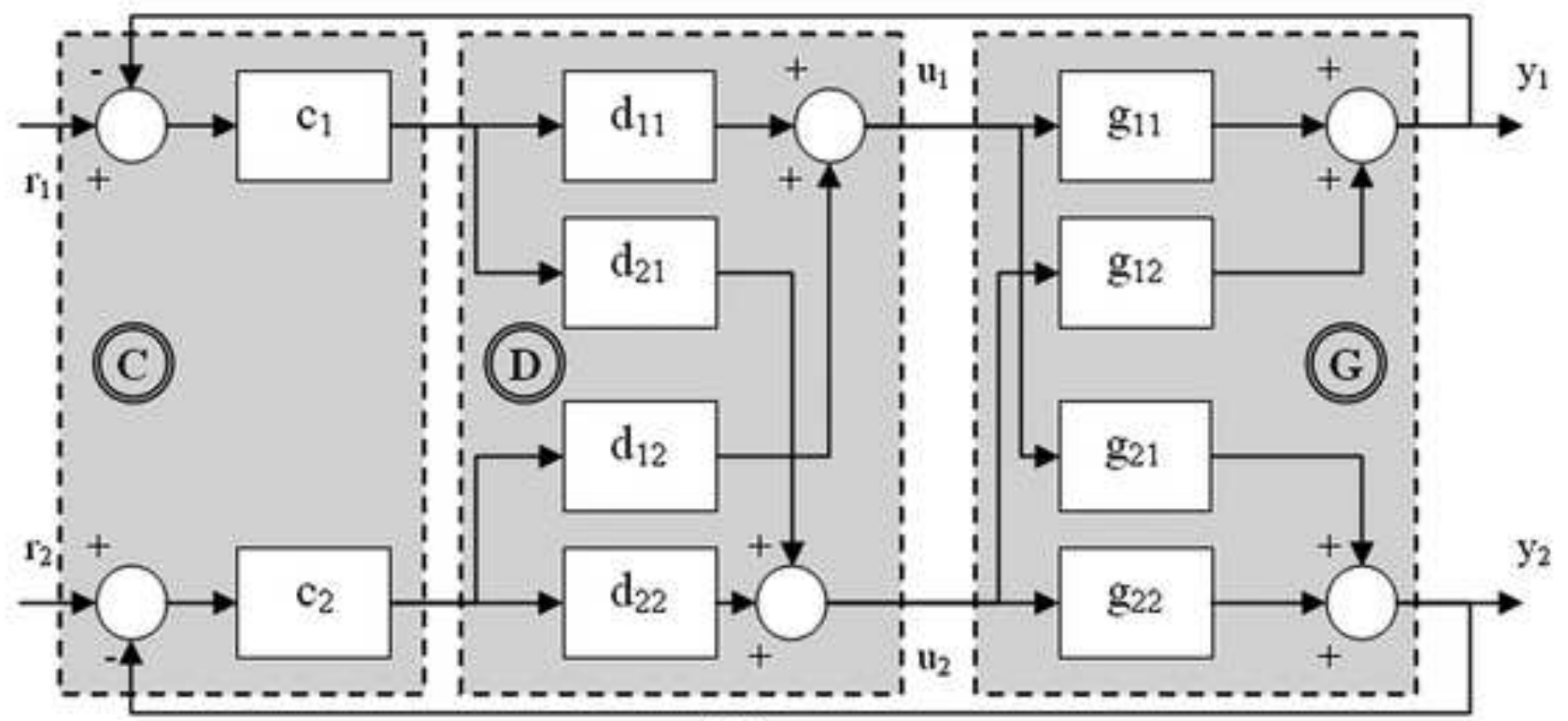

(a)

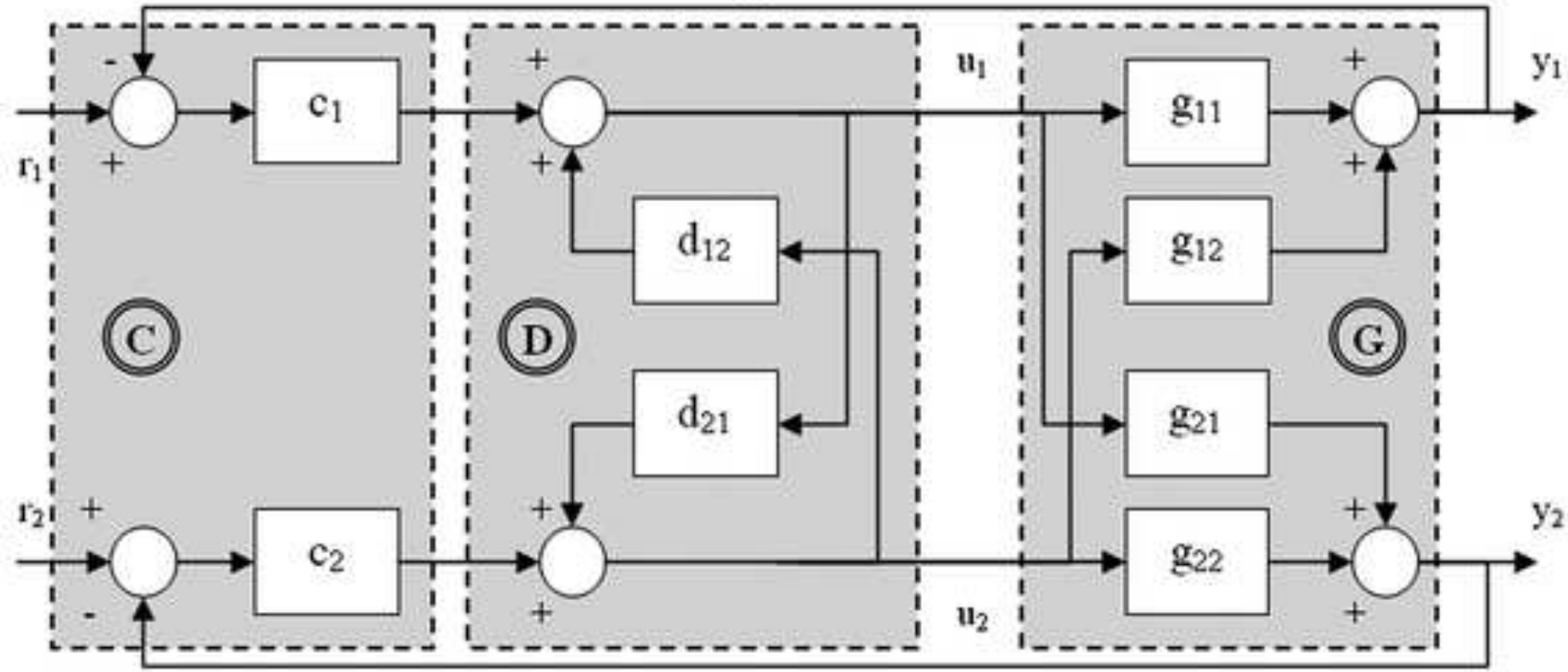

(b) 

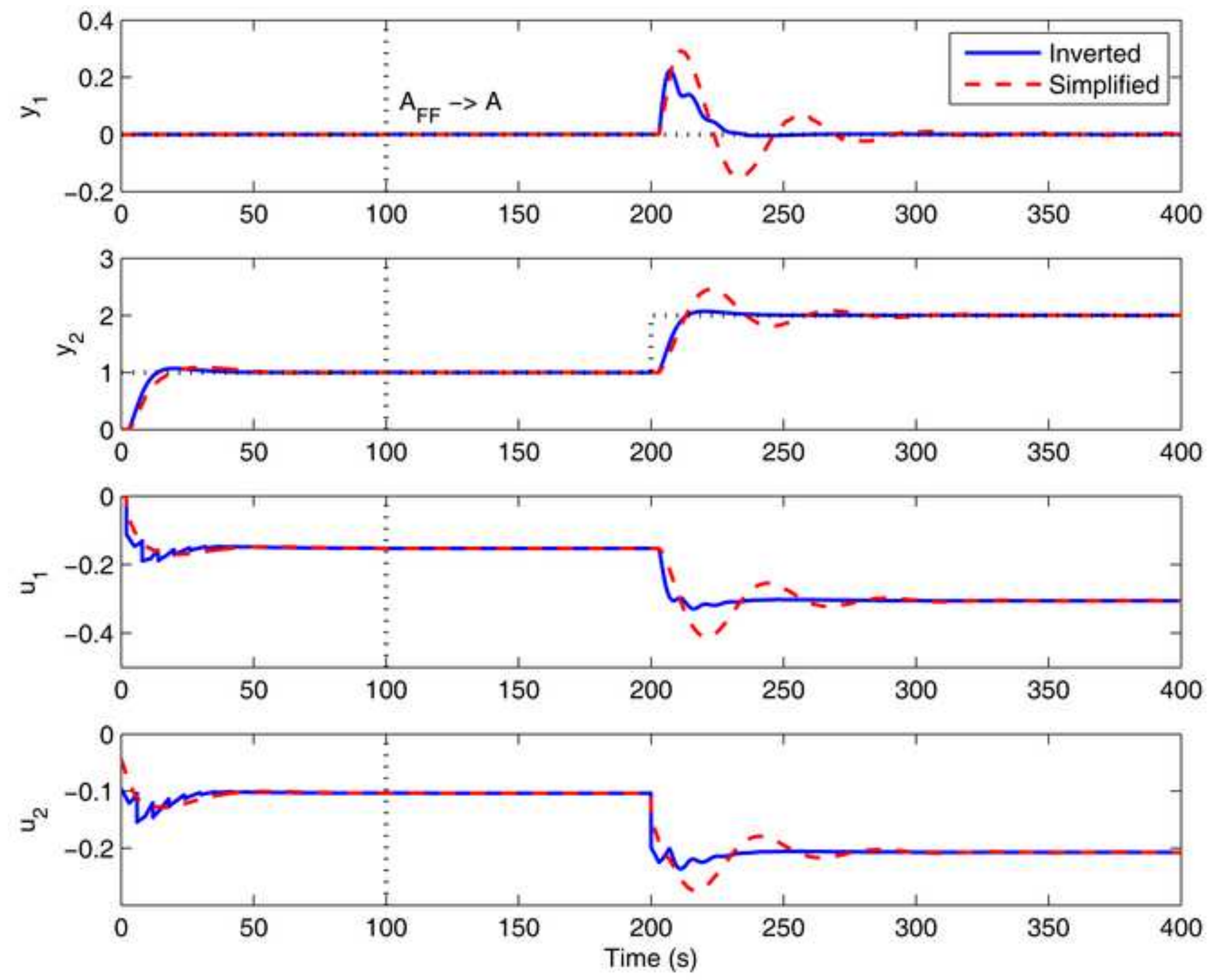


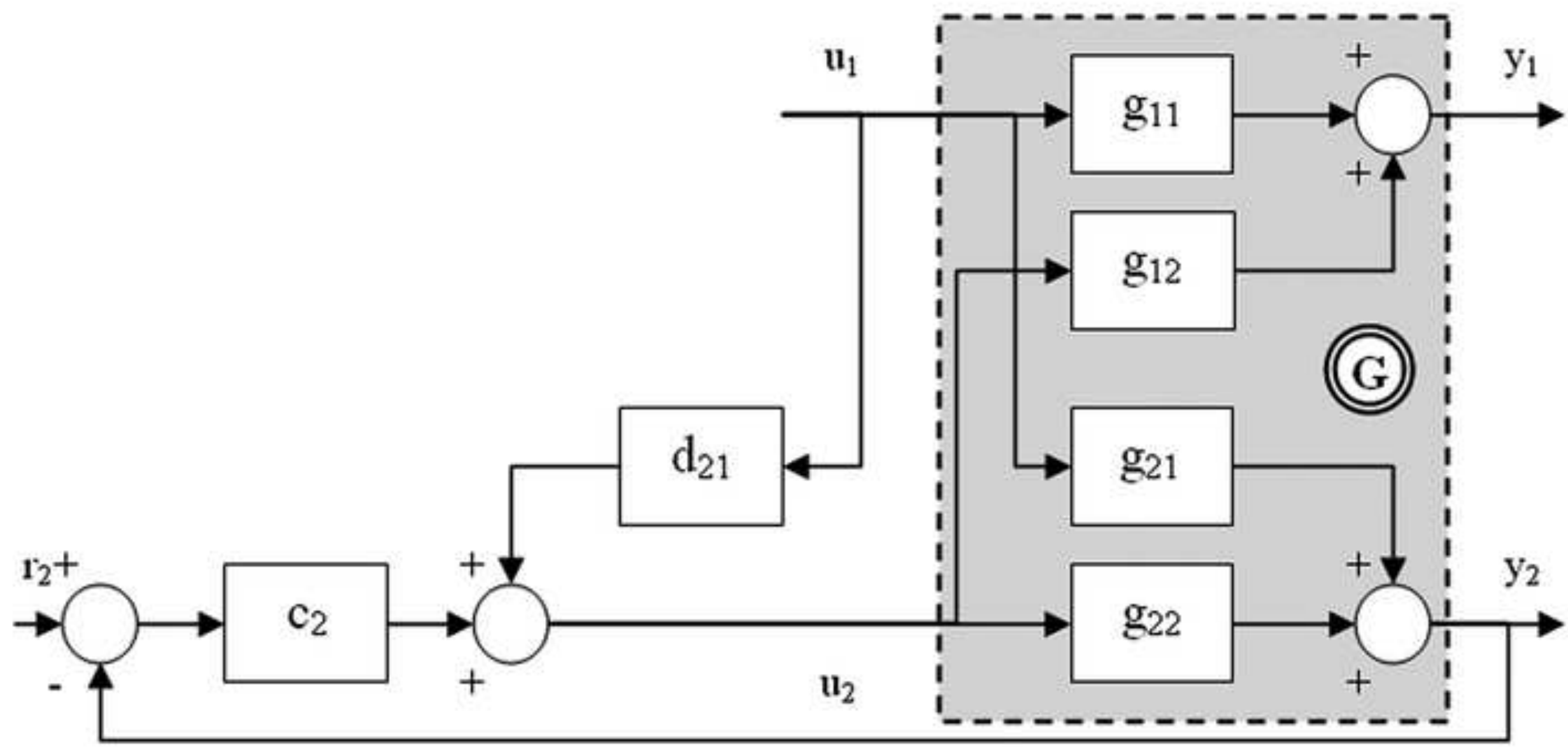



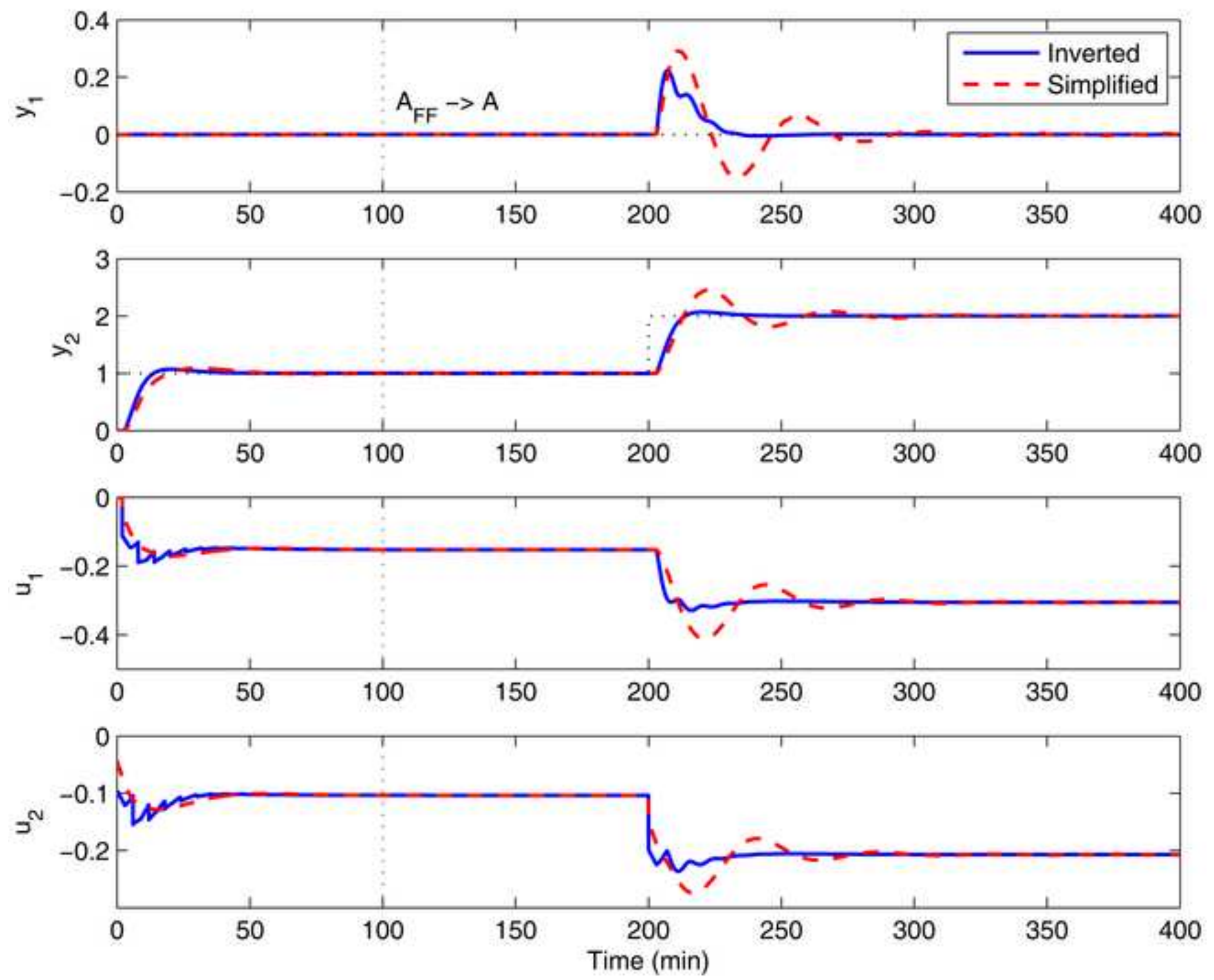

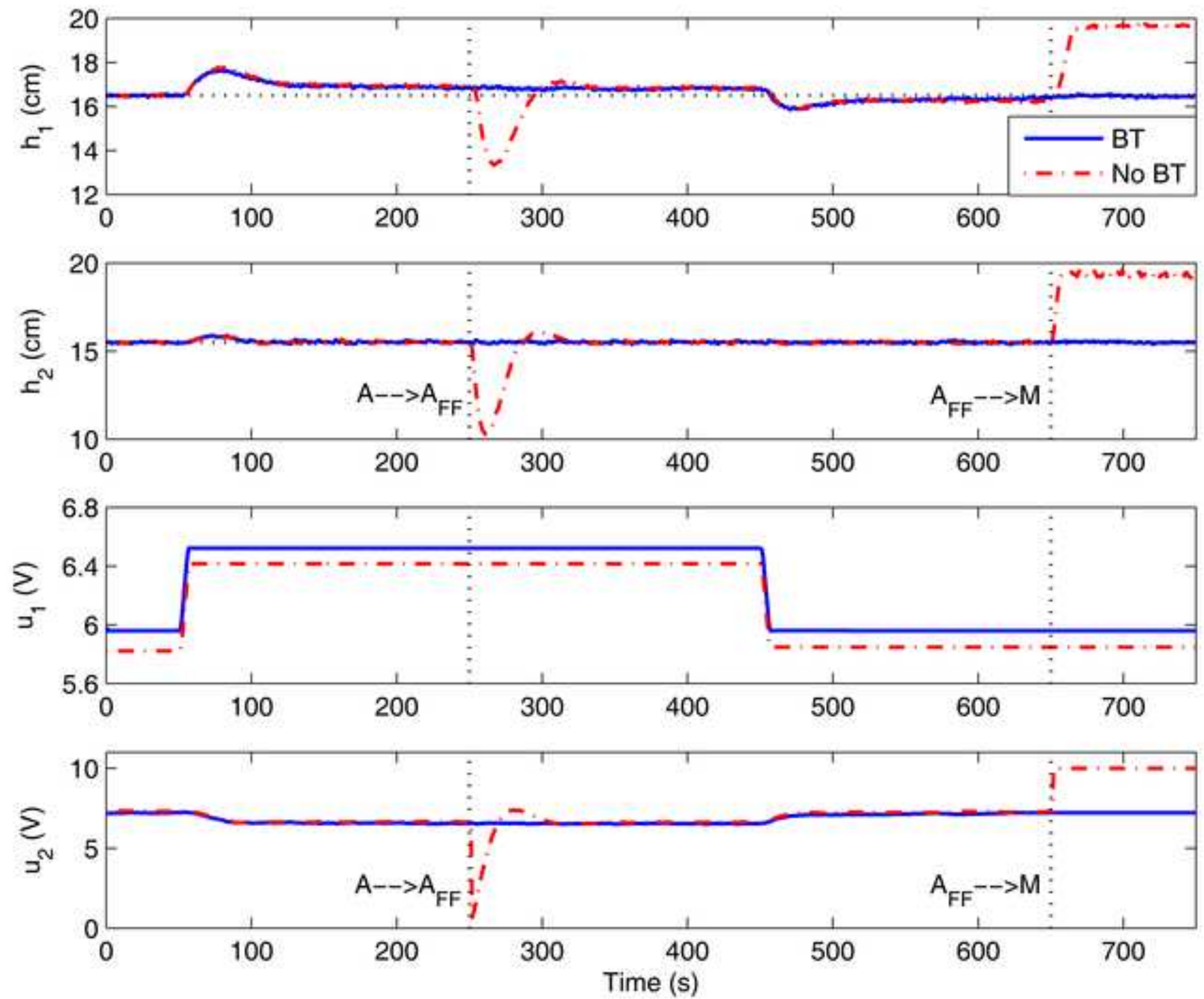


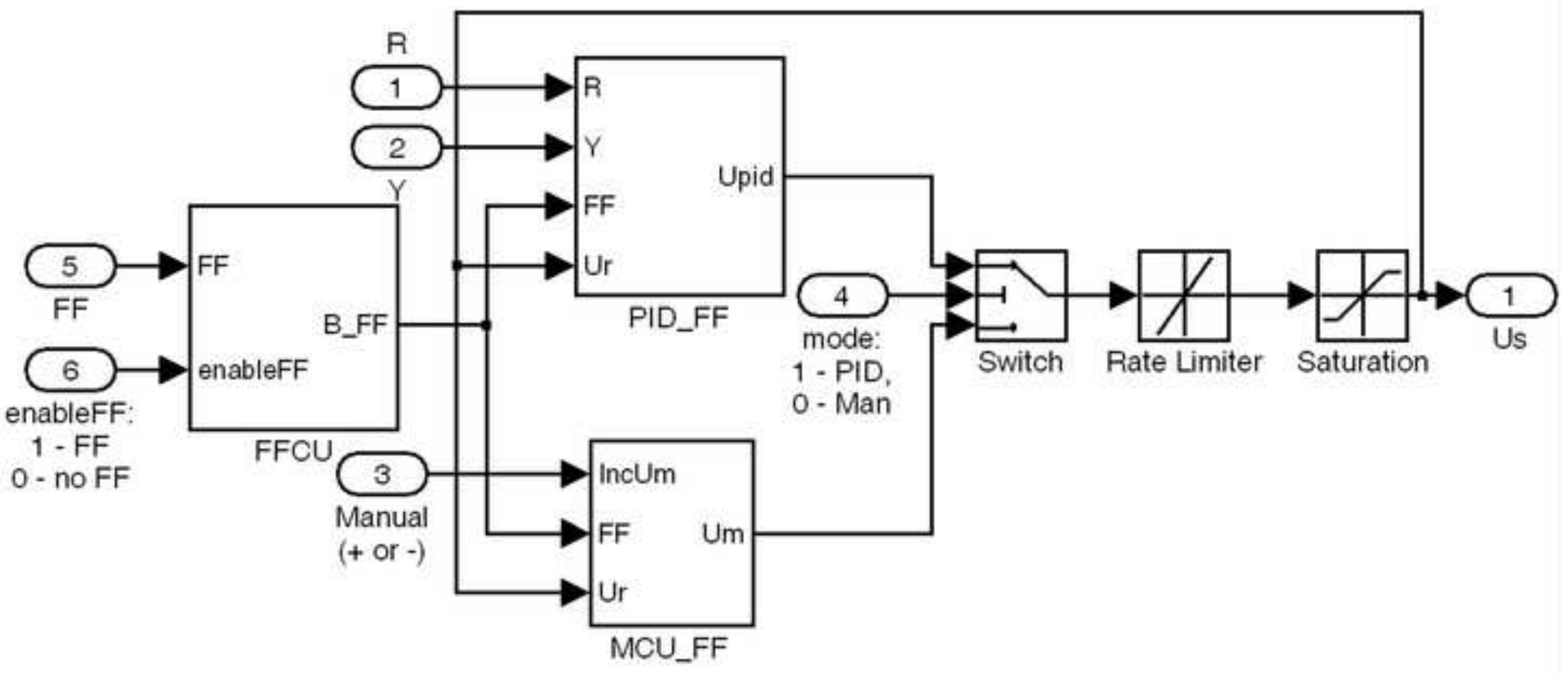



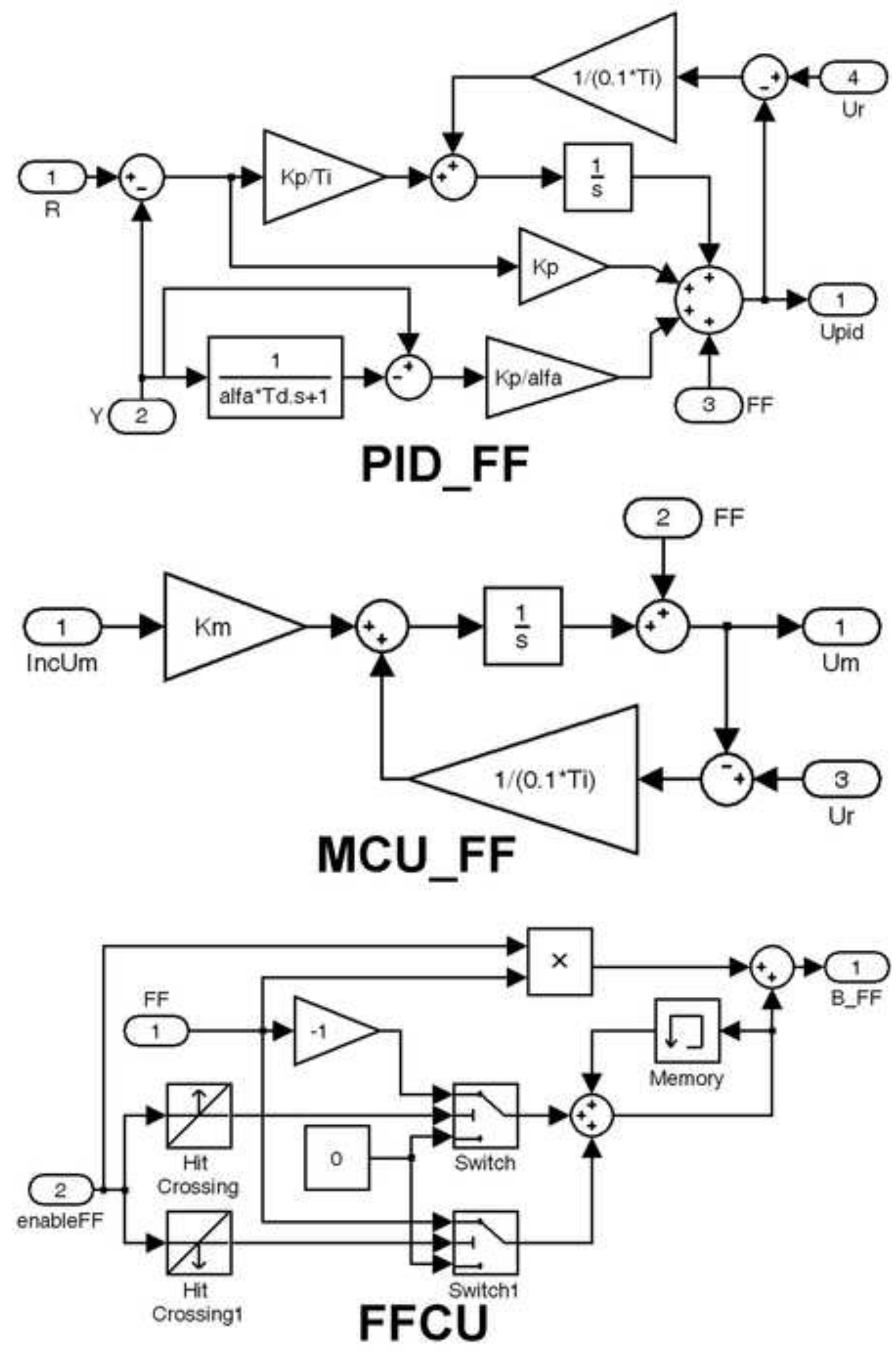


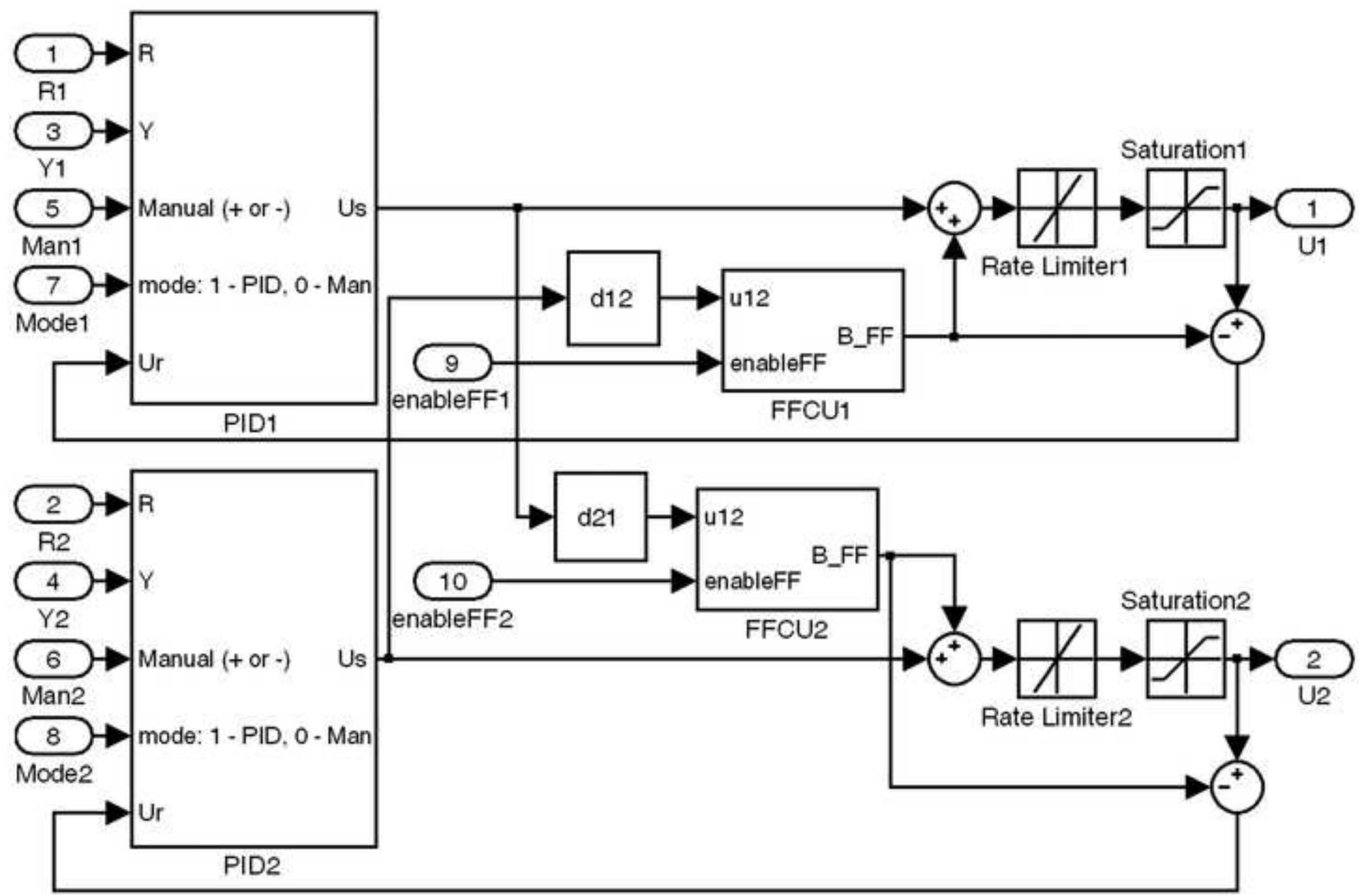



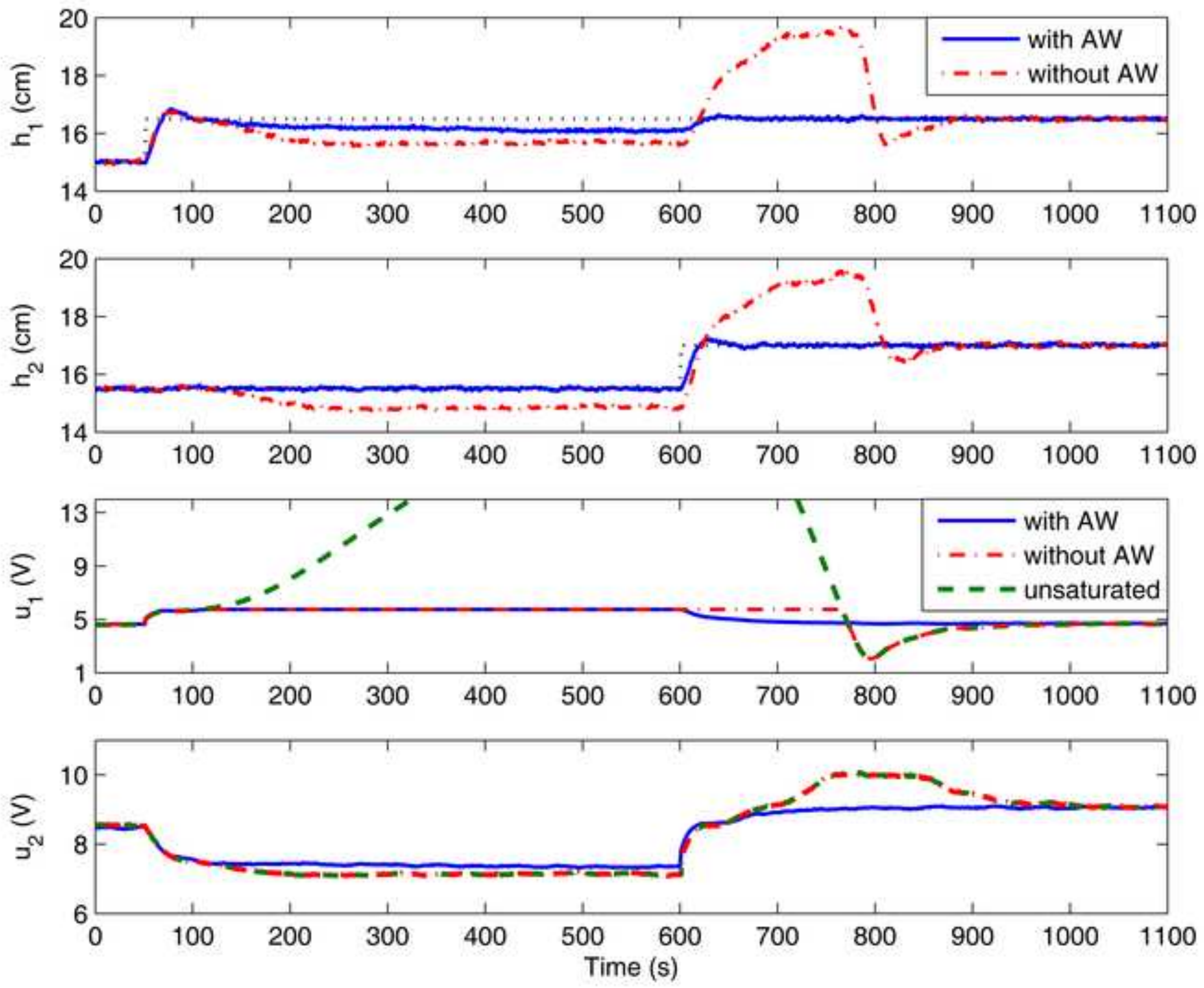

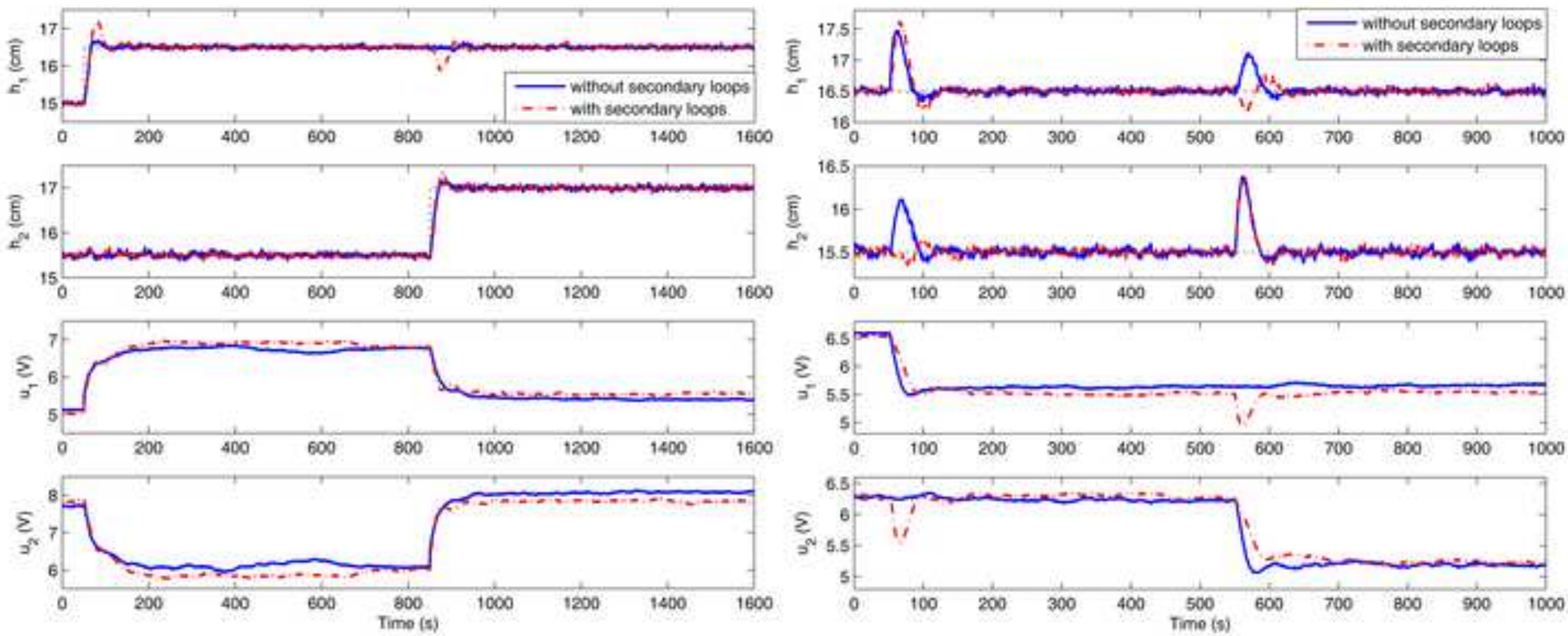

(a)

(b) 